\title{
Genomic landscape and prognosis of patients with TP53-mutated non-small cell lung cancer
}

\author{
Zhisong Fan ${ }^{1 \#}$, Qi Zhang ${ }^{1 \#}$, Li Feng ${ }^{1 \#}$, Long Wang ${ }^{1}$, Xinliang Zhou ${ }^{1}$, Jing Han ${ }^{1}$, Dan Li $^{1}$, Jiayin Liu ${ }^{1}$, \\ Xue Zhang ${ }^{1}$, Jing Zuo ${ }^{1}$, Xiao Zou ${ }^{2}$, Yiran $\mathrm{Cai}^{2}$, Ying Sun ${ }^{2}$, Yudong Wang ${ }^{1 \wedge}$ \\ ${ }^{1}$ Department of Medical Oncology, The Fourth Hospital of Hebei Medical University, Shijiazhuang, China; ${ }^{2}$ Burning Rock Biotech, Guangzhou, China \\ Contributions: (I) Conception and design: Y Wang; (II) Administrative support: Y Wang, J Zuo; (III) Provision of study materials or patients: Z Fan, \\ Q Zhang, L Feng, L Wang, X Zhou, J Han, D Li; (IV) Collection and assembly of data: Z Fan, Q Zhang, L Feng, J Liu, X Zhang, J Zuo; (V) Data \\ analysis and interpretation: Z Fan, Q Zhang, Y Wang, X Zou, Y Cai, Y Sun; (VI) Manuscript writing: All authors; (VII) Final approval of manuscript: \\ All authors. \\ "These authors contributed equally to this work. \\ Correspondence to: Yudong Wang. Department of Medical Oncology, The Fourth Hospital of Hebei Medical University, No. 12 Jiankang Road, \\ Shijiazhuang 050011, China. Email: wyd_999@126.com.
}

Background: The TP53 tumor suppressor gene plays an important role in preventing and inhibiting the growth of tumor by regulating cell cycle, apoptosis and DNA repair. Meanwhile, the TP53 gene is one of the most frequently altered gene in non-small cell lung cancer (NSCLC) patients. Mutant TP53 (TP53-MUT) may lose tumor suppressor activity and gain tumor promoting functions, which play an important role in cancer risk, therapy resistance and poor prognosis. The impact of TP53-MUT on the prognosis of NSCLC patients need to be further studied.

Methods: We obtained genomic and clinical data from The Cancer Genome Atlas (TCGA). Mutation profiles, the TMB, disease-free survival (DFS), and overall survival (OS) were compared between patients with different TP53-MUT statuses.

Results: TP53-MUTs were detected in 46.6\% of patients with lung adenocarcinoma (LUAD) (264 of 566 ) and $82.3 \%$ of those with lung squamous cell carcinoma (LUSC) (401 of 487). The most frequently comutated genes in patients with LUAD carrying a TP53-MUT included classic driver genes such as epidermal growth factor receptor $(E G F R)$ and anaplastic large-cell lymphoma kinase $(A L K)$, while Kirsten rat sarcoma viral oncogene (KRAS) mutations and TP53-MUTs appear to be mutually exclusive. This mutual exclusivity was not observed in patients with LUSC, in whom titin (TTN) and CUB and Sushi multiple domains 3 (CSMD3) were the most frequently co-mutated genes. A higher TMB was significantly associated with TP53-MUTs in patients with LUAD but not in those with LUSC. In patients with stage I-III NSCLC who had undergone surgery, there was no significant difference in DFS between patients carrying TP53-wildtype (TP53-WT) and TP53-MUTs, irrespective of histology or mutation type. However, the presence of TP53MUT was associated with shorter OS in patients with LUAD (49 vs. 54 months, respectively; $\mathrm{P}=0.13$ ) and significantly longer OS in those with LUSC (62 vs. 29 months, respectively; $\mathrm{P}=0.015$ ).

Conclusions: In contrast to most previous studies, we revealed TP53-MUT characteristic in NSCLC patients according to histology-specific differences and the association between TP53-MUT and the mutation landscape, the TMB, and the OS. These findings suggest a need for individualized management for patients with LUAD and LUSC who carry a TP53-MUT, and warrant further research.

Keywords: Non-small cell lung cancer (NSCLC); lung squamous cell carcinoma (LUSC); lung adenocarcinoma (LUAD); TP53 mutation; prognosis

^ ORCID: 0000-0001-8302-5518. 
Submitted Dec 14, 2021. Accepted for publication Feb 21, 2022.

doi: $10.21037 /$ atm-22-412

View this article at: https://dx.doi.org/10.21037/atm-22-412

\section{Introduction}

Lung cancer is a malignant tumor with the highest morbidity and mortality worldwide (1). In 2020, there were 2.2 million new cases and 1.8 million deaths related to lung cancer (1). Lung cancer is the top-ranking malignancy in incidence and mortality in China (2). Nonsmall cell lung cancer (NSCLC) is the most common subtype, accounting for $83 \%$ of all lung cancer cases (3). Identification of oncogenic driver alterations in recent decades has accelerated the development of targeted therapy for NSCLC. Clinically actionable driver alterations are frequently observed in protein kinase-encoding genes such as epidermal growth factor receptor $(E G F R)$ and anaplastic lymphoma kinase $(A L K)$. In addition, drugs are being actively developed to target other drivers, such as Kirsten rat sarcoma viral oncogene (KRAS) and phosphatidylinositol 3-kinase catalytic subunit $\alpha$ (PIK3CA).

In addition to proto-oncogene activation, aberrant tumor suppressors constitute a significant type of oncogenic alterations, the most frequent of which is tumor protein 53 (TP53). TP53 is located on chromosome $17 \mathrm{p}$ and encodes p53 (4,5). A mutant p53 loses its normal grip on the regulation of cell growth, apoptosis, and DNA repair, thereby promoting tumor initiation and growth (5). Almost $80 \%$ of TP53 mutations (TP53-MUTs) are missense mutations, the rest including frameshifts, truncations, and deletions (5).

TP53-MUTs are more likely occurred in smoking NSCLC patients (6). Previous research suggests that different classes of TP53-MUTs, based on type and location, may have different prognostic significance (7-10). However, evidence is still accumulating regarding the association between TP53-MUTs and clinical features, co-mutations, and prognosis in NSCLC. Moreover, most studies have investigated NSCLC patients as a homogeneous population or focused mainly on lung adenocarcinoma (LUAD), leaving lung squamous cell carcinoma (LUSC) as an under-characterized subtype. To determine the prognostic relevance of TP53-MUTs in patients with LUAD and LUSC, we retrospectively analyzed data from The Cancer Genome Atlas (TCGA) database and identified differences in the mutational landscape, tumor mutation burden (TMB), disease-free survival (DFS), and overall survival (OS).

We present the following article in accordance with the REMARK reporting checklist (available at https://atm. amegroups.com/article/view/10.21037/atm-22-412/rc).

\section{Methods}

\section{Data and patients}

Genomic and clinical data for patients with NSCLC were obtained from TCGA, a publicly available database at http://www.cbioportal.org. The study was conducted in accordance with the Declaration of Helsinki (as revised in 2013). The study cohort consisted of 1,053 patients, including 566 patients with LUAD and 487 with LUSC. Only the 931 patients with stage I-III NSCLC who underwent surgery were included for subsequent survival analyses, including 470 patients with LUAD and 461 with LUSC. OS was defined as the time interval between the date of tumor biopsy and the date of death. DFS was defined as the time from the day of surgery to disease progression or the last follow-up.

Four different binary systems were used to classify TP53MUTs. First, they were classified as loss-of-function (LOF) or non-LOF mutations (7). The former group included nonsense and frameshift mutations that significantly disrupted $\mathrm{p} 53$ translation and caused LOF. The remaining mutations were classified in the non-LOF group. Secondly, mutations were divided into hot exon (exons 5-8) and nonhot exon (other exons) groups, according to whether or not the mutation was located on exons 5-8, which encode the DNA-binding domain and harbor most TP53-MUTs (8). The third system distinguished between disruptive and non-disruptive mutations. Disruptive mutations included terminating mutations and substitutions within the L2 or L3 binding domains with codons of amino acids of a different polarity or charge group (9). Disruptive mutations may lead to complete or almost complete p53 LOF, while non-destructive mutants can retain some functions. The final binary system classified patients into EAp53 highrisk (EAp53 score $\geq 75$ ) and EAp53 low-risk groups (EAp53 score $<75$ ), in which EAp53 referred to the evolutionary 
Table 1 Clinicopathological characteristics of patients with NSCLC

\begin{tabular}{|c|c|}
\hline Characteristics & Number (\%) \\
\hline \multicolumn{2}{|l|}{ Subtype } \\
\hline LUAD & $566(53.8)$ \\
\hline LUSC & $487(46.2)$ \\
\hline \multicolumn{2}{|l|}{ Gender } \\
\hline Female & $402(38.2)$ \\
\hline Male & $597(56.7)$ \\
\hline NA & $54(5.1)$ \\
\hline \multicolumn{2}{|l|}{ TNM stage } \\
\hline I & $514(48.8)$ \\
\hline II & $282(26.8)$ \\
\hline III & $166(15.8)$ \\
\hline IV & $34(3.2)$ \\
\hline NA & $57(5.4)$ \\
\hline \multicolumn{2}{|l|}{ Received neoadjuvant therapy } \\
\hline No & $991(94.1)$ \\
\hline Yes & $8(0.6)$ \\
\hline NA & $54(5.1)$ \\
\hline \multicolumn{2}{|l|}{ Received radiation therapy } \\
\hline No & $776(73.7)$ \\
\hline Yes & $113(10.7)$ \\
\hline NA & $164(15.6)$ \\
\hline \multicolumn{2}{|l|}{ Tumor diagnosis } \\
\hline Recrudescence & $547(52.0)$ \\
\hline New diagnosis & $292(27.7)$ \\
\hline NA & $214(20.3)$ \\
\hline \multicolumn{2}{|l|}{ Race } \\
\hline American Indian or Alaska Native & $1(0.1)$ \\
\hline Asian & $17(1.6)$ \\
\hline Black or African American & $81(7.7)$ \\
\hline White & $725(68.9)$ \\
\hline NA & $229(21.8)$ \\
\hline \multicolumn{2}{|l|}{ Survival } \\
\hline No & $396(37.6)$ \\
\hline Yes & $605(57.5)$ \\
\hline NA & $52(4.9)$ \\
\hline
\end{tabular}

NSCLC, non-small cell lung cancer; LUAD, lung adenocarcinoma; LUSC, lung squamous cell carcinoma. action score of the TP53 missense mutations (10). Higher scores indicated more harmful mutations (10). As EAp53 annotations could only be automated for approximately $50 \%$ of the TP53-MUTs in the dataset, the remaining mutations were annotated manually. In these cases, terminating, and frameshift mutations were defined as highrisk, and splice region mutations as low-risk.

\section{Statistical analysis}

Unpaired $t$-tests were used to compare continuous variables between the different binary groups. A Chi-squared test was used to compare the proportions of categorical variables between groups. Survival was illustrated using KaplanMeier curves, with $\mathrm{P}$ values determined by log-rank tests, and the hazard ratio (HR) and $95 \%$ confidence interval (CI) were determined by Cox regression models. All statistical tests were 2-tailed and were conducted in $\mathrm{R}$ (version 3.4.2, The R Foundation, https://www.r-project.org/). A 2-tailed $\mathrm{P}$ value of $<0.05$ was considered statistically significant.

\section{Results}

\section{Clinical samples and histopathologic data}

The baseline clinicopathological characteristics of the study cohort are shown in Tables 1,2. Of the 1,053 patients with NSCLC, 566 had LUAD and 487 had LUSC. Among them, 597 were male and 402 were female. Most patients did not receive neoadjuvant therapy $(94.1 \%)$ or radiotherapy (73.7\%). A cohort of patients with stage I-III NSCLC who underwent surgical treatment $(\mathrm{n}=931)$ was used to analyze survival time. This cohort consisted of 470 patients with LUAD and 461 with LUSC. Like the 1,053-patient cohort, most of these patients did not receive neoadjuvant therapy $(99.1 \%)$ or radiotherapy $(80.0 \%)$.

\section{Rate and distribution of different classes of TP53-MUT}

Of the patients with NSCLC, 63.2\% (665 of 1,053) carried a TP53-MUT. The TP53-MUT rate was significantly lower in patients with LUAD $(46.6 \%, 264$ of 566) than in those with LUSC (82.3\%, 401 of 487). No significant difference was observed in the distribution of TP53-MUTs between patients with LUAD and LUSC (Figure 1). In the four different binary systems, the LOF group was identified in $37.0 \%$ of patients with LUAD and $33.5 \%$ of those with LUSC $(\mathrm{P}=0.35)$; the hot exon group was identified in $72.5 \%$ of patients with LUAD and $76.1 \%$ with LUSC $(\mathrm{P}=0.30)$; 


\section{Page 4 of 12}

Table 2 Clinicopathological characteristics of patients with stage IIII NSCLC used for survival analysis

\begin{tabular}{|c|c|c|c|}
\hline Characteristics & LUAD (\%) & LUSC (\%) & Overall \\
\hline Total & $470(50.5)$ & $461(49.5)$ & 931 \\
\hline \multicolumn{4}{|l|}{ Gender } \\
\hline Female & $257(67.3)$ & $125(32.7)$ & 382 \\
\hline Male & $213(38.8)$ & $336(61.2)$ & 549 \\
\hline \multicolumn{4}{|l|}{ TNM stage } \\
\hline I & $271(54.1)$ & $230(45.9)$ & 501 \\
\hline II & $119(44.2)$ & $150(55.8)$ & 269 \\
\hline III & $80(49.7)$ & $81(50.3)$ & 161 \\
\hline \multicolumn{4}{|l|}{ T stage } \\
\hline $\mathrm{T} 1$ & $159(60.5)$ & $104(39.5)$ & 263 \\
\hline $\mathrm{T} 2$ & $255(48.7)$ & 269 (51.3) & 524 \\
\hline T3 & $43(39.1)$ & $67(60.9)$ & 110 \\
\hline $\mathrm{T} 4$ & $13(38.2)$ & $21(61.8)$ & 34 \\
\hline \multicolumn{4}{|l|}{$\mathrm{N}$ stage } \\
\hline NO & $310(51.4)$ & $293(48.6)$ & 603 \\
\hline N1 & $87(42.2)$ & $119(57.8)$ & 206 \\
\hline N2 & $65(62.5)$ & $39(37.5)$ & 104 \\
\hline N3 & $2(28.6)$ & $5(71.4)$ & 7 \\
\hline NX & $6(54.5)$ & $5(45.5)$ & 11 \\
\hline \multicolumn{4}{|c|}{ Neoadjuvant therapy } \\
\hline No & $467(50.6)$ & $456(49.4)$ & 923 \\
\hline Yes & $3(37.5)$ & $5(62.5)$ & 8 \\
\hline \multicolumn{4}{|l|}{ Radiation therapy } \\
\hline No & 377 (51.9) & $349(48.1)$ & 726 \\
\hline Yes & $52(50.5)$ & $51(49.5)$ & 103 \\
\hline NA & $41(40.2)$ & $61(59.8)$ & 102 \\
\hline
\end{tabular}

LUAD, lung adenocarcinoma; LUSC, lung squamous cell carcinoma.

the disruptive group was identified in $50.7 \%$ of patients with LUAD and $48.8 \%$ with LUSC $(\mathrm{P}=0.65)$; and the EAp53 high-risk group was identified in $74.8 \%$ of patients with LUAD and $75.6 \%$ with LUSC $(\mathrm{P}=0.86)$.

\section{Co-mutations}

Most classic LUAD genomic alterations occurred more

\section{Fan et al. The prognosis of NSCLC patients with TP53-mutation}

frequently in patients carrying TP53-MUTs than in those carrying the TP53-wildtype (TP53-WT) (Figure 2A): EGFR, $16.1 \%$ vs. $7.3 \%$, respectively $(\mathrm{P}=0.001) ; A L K, 8.6 \%$ vs. $3.6 \%$, respectively $(\mathrm{P}=0.02)$; v-raf murine sarcoma viral oncogene homolog B1 $(B R A F), 10.4 \%$ vs. $5.0 \%$, respectively $(\mathrm{P}=0.02)$; and rearranged during transfection (RET), 6.8\% vs. $1.0 \%$, respectively $(\mathrm{P}=0.001)$. On the other hand, in patients with LUAD, KRAS mutations were less frequent among patients carrying a TP53-MUT than in those carrying the TP53-WT (21.8\% vs. 33.7\%, respectively; $\mathrm{P}=0.002$ ) (Figure $2 B$ ). Specifically, patients carrying a TP53MUT were significantly less likely to carry KRAS G12X than those carrying the TP53-WT (17.9\% vs. $31.4 \%$, respectively; $\mathrm{P}<0.001$ ) (Figure $2 C$ ). Within the 8 classic NSCLC-associated driver genes and the 14 most frequently altered genes in patients with LUAD, the mutation rates of most genes were not significantly different when stratified according to the 4 TP53-MUT classification systems (Figure S1). Only mucin 16 (MUC16) was significantly more frequently altered among patients in the EAp53 highrisk group than among those in the low-risk group (58.0\% vs. $41.2 \%$, respectively; $\mathrm{P}=0.022$ ) (Figure $\mathrm{S} 1$ ).

In patients with LUSC, the top 5 most frequently comutated genes were titin (TTN), CUB and Sushi multiple domains 3 (CSMD3), the type 2 ryanodine receptor (RYR2), low-density lipoprotein receptor-related protein 1B (LRP1B) and usher syndrome type IIA (USH2A). Their mutated rates were significantly higher in patients carrying a TP53MUT than in those carrying the TP53-WT: TTN $(78.7 \%$ vs. 44.6\%; $\mathrm{P}<0.01$ ), CSMD3 (48.4\% vs. $21.7 \% ; \mathrm{P}<0.01$ ), RYR2 (42.6\% vs. $26.5 \% ; \mathrm{P}=0.009)$, LRPB1 (38.8\% vs. $18.1 \% ; \mathrm{P}<0.01$ ), and USH 2 A (35.3\% vs. $22.9 \% ; \mathrm{P}=0.038$; Figure 2D). Subgroup analysis according to the TP53-MUT type showed that among the 25 most common genes in patients with LUSC, the mutation rates in patients carrying hot exon TP53-MUTs were significantly higher than in those with non-hot exon mutations for LRPB1 (41.6\% vs. $30.1 \%$, respectively; $\mathrm{P}=0.049)$ and synaptic nuclear envelope protein 1 (SYNE1) (34.6\% vs. $23.3 \%$, respectively; $\mathrm{P}=0.044)$. In addition, $U S H 2 A$ was significantly more frequently altered in patients with non-disruptive TP53MUTs than in those with disruptive TP53-MUTs (41.1\% vs. 28.6\%, respectively; $\mathrm{P}=0.009$ ), and in patients in the EAp53 low-risk group compared to those in the EAp53 high-risk group (44.6\% vs. $32.5 \%$, respectively; $\mathrm{P}=0.036$ ). The profiles of frequent alterations co-occurring with each type of TP53-MUT in patients with LUSC are presented in Figure $\mathrm{S} 1$. 

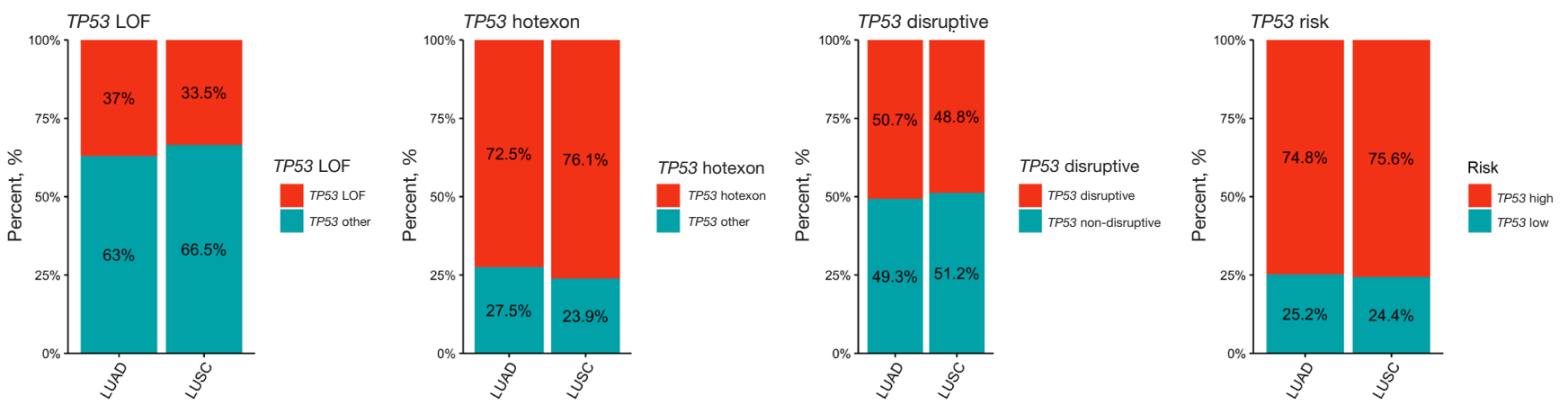

Figure 1 Percentages of patients with lung adenocarcinoma (LUAD) and squamous cell carcinoma (LUSC) carrying different classes of TP53 mutations.
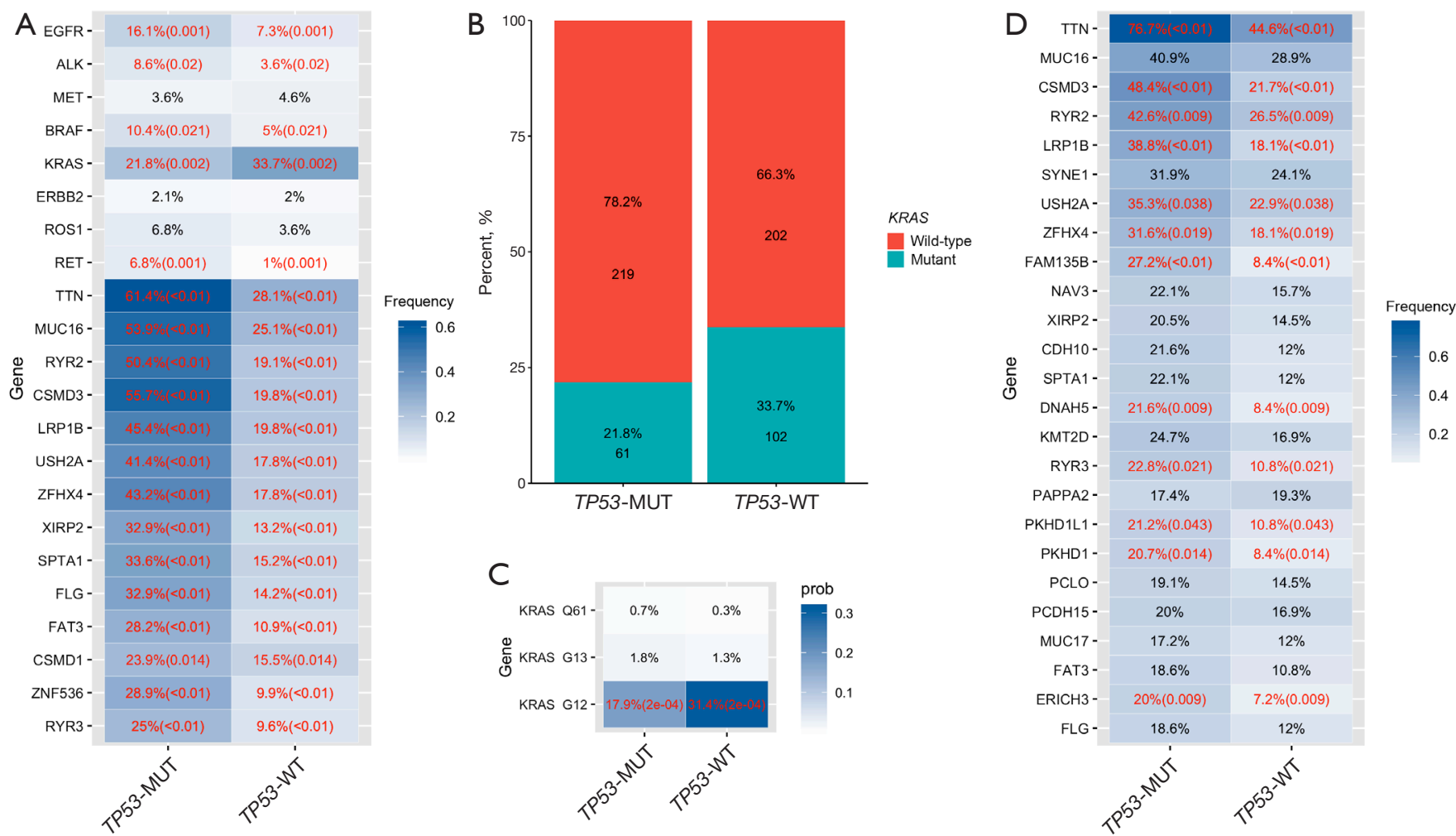

Figure 2 Differences of mutant genes between TP53 mutant (TP53-MUTs) group and wild-type (TP53-WT) group in non-small cell lung cancer (NSCLC). (A) Mutation rates of 8 classic oncogenes in NSCLC and the 14 most frequently mutated genes in patients with lung adenocarcinoma (LUAD) carrying TP53-MUTs compared with TP53-WT. Significantly different rates (shown in red) are followed by the corresponding $\mathrm{P}$ values in parentheses; (B) mutation rates of Kirsten rat sarcoma viral oncogene (KRAS) in patients with LUAD carrying TP53-WT and TP53-MUTs; (C) mutation rates at the 3 most common KRAS hotspot codons in patients with LUAD; (D) mutation rates of the most frequently mutated genes in patients with lung squamous cell carcinoma (LUSC) carrying TP53-WT or a TP53-MUT.

\section{Association between TP53-MUTs and the TMB}

Overall, patients with NSCLC who carried a TP53-MUT showed a significantly higher TMB than those carrying the TP53-WT (6.0 vs. 2.6 mut/Mb, respectively; $\mathrm{P}<0.001$ )

(Figure 3A). A similar distinction was observed in patients with LUAD, that patients carrying TP53-MUTs had a 

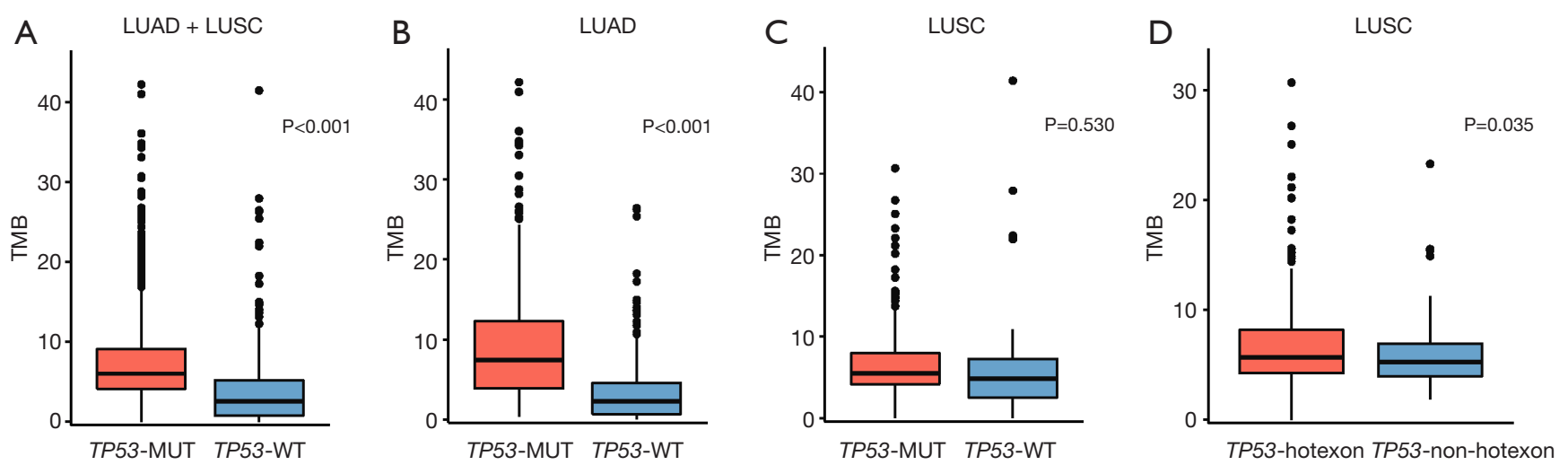

Figure 3 The tumor mutation burden (TMB) of patients carrying TP53-wild type (TP53-WT) and TP53 mutations (TP53-MUTs) in (A) the entire cohort, (B) the lung adenocarcinoma (LUAD) subgroup, and (C) the lung squamous cell carcinoma (LUSC) subgroup. (D) TMB levels of patients with LUSC harboring TP53 hot and non-hot exon mutations.

significantly higher TMB than those carrying the TP53WT (7.4 vs. 2.3 mut/Mb, respectively; $\mathrm{P}<0.001$ ) (Figure $3 B$ ), but the distinction was not observed in patients with LUSC (5.5 vs. 4.8 mut $/ \mathrm{Mb}$, respectively; $\mathrm{P}=0.53$ ) (Figure 3C). In patients with LUSC carrying a TP53-MUT, subgroup analysis showed that the hot exon group had a significantly higher TMB than the non-hot exon group (5.7 vs. 5.2 mut $/ \mathrm{Mb}$, respectively; $\mathrm{P}=0.035$ ) (Figure $3 D$ ). No significant difference was observed in pairwise comparisons of TMB levels in other TP53-MUT classifications for patients with either LUAD or LUSC (Figure S2).

\section{Prognostic significance of different types of TP53-MUT in patients with stage I-III NSCLC who underwent surgery}

Survival analysis of the 931 patients with stage I-III cancer who underwent surgery showed no significant difference in DFS between patients carrying the TP53-WT and those carrying a TP53-MUT, irrespective of the histologic subtype (Figures S3,S4). Subgroup analysis revealed no significant difference in DFS for patients with LUAD or LUSC who carried a TP53-MUT when stratified by any of the 4 TP53-MUT classification systems (Figures S3,S4).

In terms of OS, for patients with LUAD, no significant difference was found between patients carrying a TP53MUT and those carrying the TP53-WT (49 vs. 54 months, respectively; $\mathrm{P}=0.13$ ) (Figure $4 A$ ). In addition, we analyzed the prognostic relevance of the different types of TP53MUTs. None of the 4 classes could significantly predict OS in patients with LUAD (Figure 4B-4E). However, in patients with LUSC, the OS was significantly longer for patients carrying a TP53-MUT than those carrying the TP53WT (62 vs. 29 months, $\mathrm{P}=0.015$; Figure $5 A$ ). Patients with LUSC carrying a non-LOF ( $\mathrm{P}=0.014)$, hot exon $(\mathrm{P}=0.017)$, disruptive $(\mathrm{P}=0.0098)$, or EAp53 high-risk $(\mathrm{P}=0.0057)$ type of TP53-MUT all had longer OS than those carrying the TP53-WT (Figure 5B-5E). But no survival difference was observed between the counterpart groups of the four type methods. Multivariate analysis showed that a TP53MUT was not an independent prognostic factor for OS in patients with stage I-III LUAD ( $\mathrm{HR}=0.8,95 \% \mathrm{CI}$ : $0.57-1.1, \mathrm{P}=0.192$ ) (Figure S5), but was a good independent prognostic factor for OS in patients with stage I-III LUSC (HR $=0.65,95 \%$ CI: $0.44-0.95, \mathrm{P}=0.028$; Figure 6).

\section{Discussion}

TP53 is the most frequently altered gene in NSCLC, occurring in $35-55 \%$ of cases. Aberrant TP53 is more prevalent in LUSC $(\sim 81 \%)$ than in LUAD $(\sim 46 \%)$ (11-13). The prevalence rate of TP53-MUTs in this study was $63.2 \%$ in NSCLC, $82.3 \%$ in LUSC, and $46.6 \%$ in LUAD. However, TP53-MUT rates were comparable within each classification systems (LOF $v s$. non-LOF, hot exon $v s$. non-hot exon, disruptive $v s$. non-disruptive, and EAp53 high-risk vs. EAp53 low-risk). There was a significant difference in the mutational landscape of TP53MUTs in patients with LUAD and LUSC. Patients with LUAD carrying a TP53-MUT were more likely to harbor aberrant classic oncogenes such as EGFR, $A L K, B R A F$, and RET, whereas patients with LUSC carrying a TP53-MUT were more likely to harbor concurrent TTN, CSMD, RYR2, 

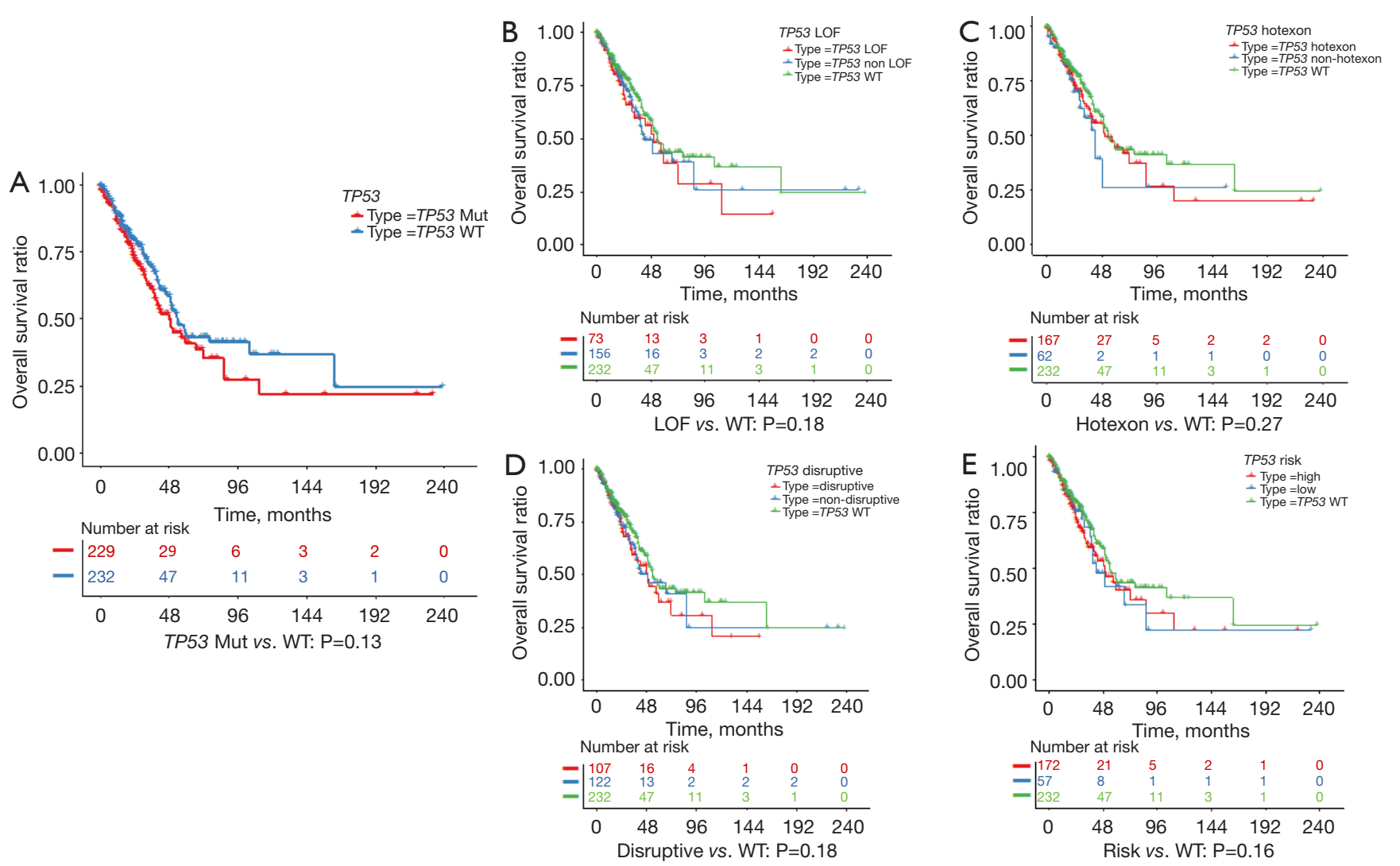

Figure 4 Kaplan-Meier survival plots showing the overall survival (OS) of patients with lung adenocarcinoma (LUAD), stratified by (A) TP53 mutation (TP53-MUT) status or (B-E) different classes of TP53-MUTs.

$L R P 1 B$, and DNAH5 mutations. Impacting carcinogenicity, co-mutations have become the core determinants in molecular and clinical heterogeneity of oncogene-driven NSCLC (14). TP53-MUT could be found in $30-72 \%$ of EGFR-mutated NSCLCs and 25-56\% of $A L K$-positive NSCLCs (15). And EGFR mutation could be found in $10 \%$ of NSCLCs with TP53-MUT (16). No matter in patients with EGFR exon 19/21 or non-exon 19/21 mutation, TP53MUT rate was higher than EGFR wild type patients (17). Co-mutated EGFR or ALK in patients carrying a TP53MUT has been associated with a reduced response to tyrosine kinase inhibitors (TKIs), most likely because of increased genomic instability due to the aberrant TP53 (15-18). On the other hand, TP53-MUT appeared to be mutually exclusive with mutations in certain genes, such as $K R A S$, and in particular with the hotspot mutation G12X. There is evidence associating KRAS and TP53 co-mutation with poor clinical outcomes in patients with NSCLC, however, these patients may derive greater benefits from anti-PD-1/PD-L1 immunotherapy than patients who do not harbor TP53 or KRAS mutation $(19,20)$. TP53-MUTs can increase the expression of immune checkpoint proteins and activate the T-effector and interferon- $\gamma$ signature. When both TP53 and KRAS are altered, the expression of PD-L1 and the TMB increases significantly (19).

Several clinical studies have suggested a positive correlation between the survival of immunotherapy and the TMB of tumor (21-24). We found a significantly higher TMB in patients with LUAD carrying a TP53-MUT than in their TP53-WT-carrying counterparts. Some researchers have consistently found an increased proportion of TP53MUTs in high TMB groups compared to low-to-medium TMB groups (25), as well as an increased TMB in patients carrying TP53-MUTs compared to those carrying TP53WT $(26,27)$. The stronger correlation between the TMB and TP53-MUTs in larger tumors, implicating the TMB and TP53 in promoting tumor growth (27). Since the TMB is considered a powerful potential biomarker for immune checkpoint inhibitors, TP53-MUTs could contribute to predicting the benefits of immune checkpoint blockades. 


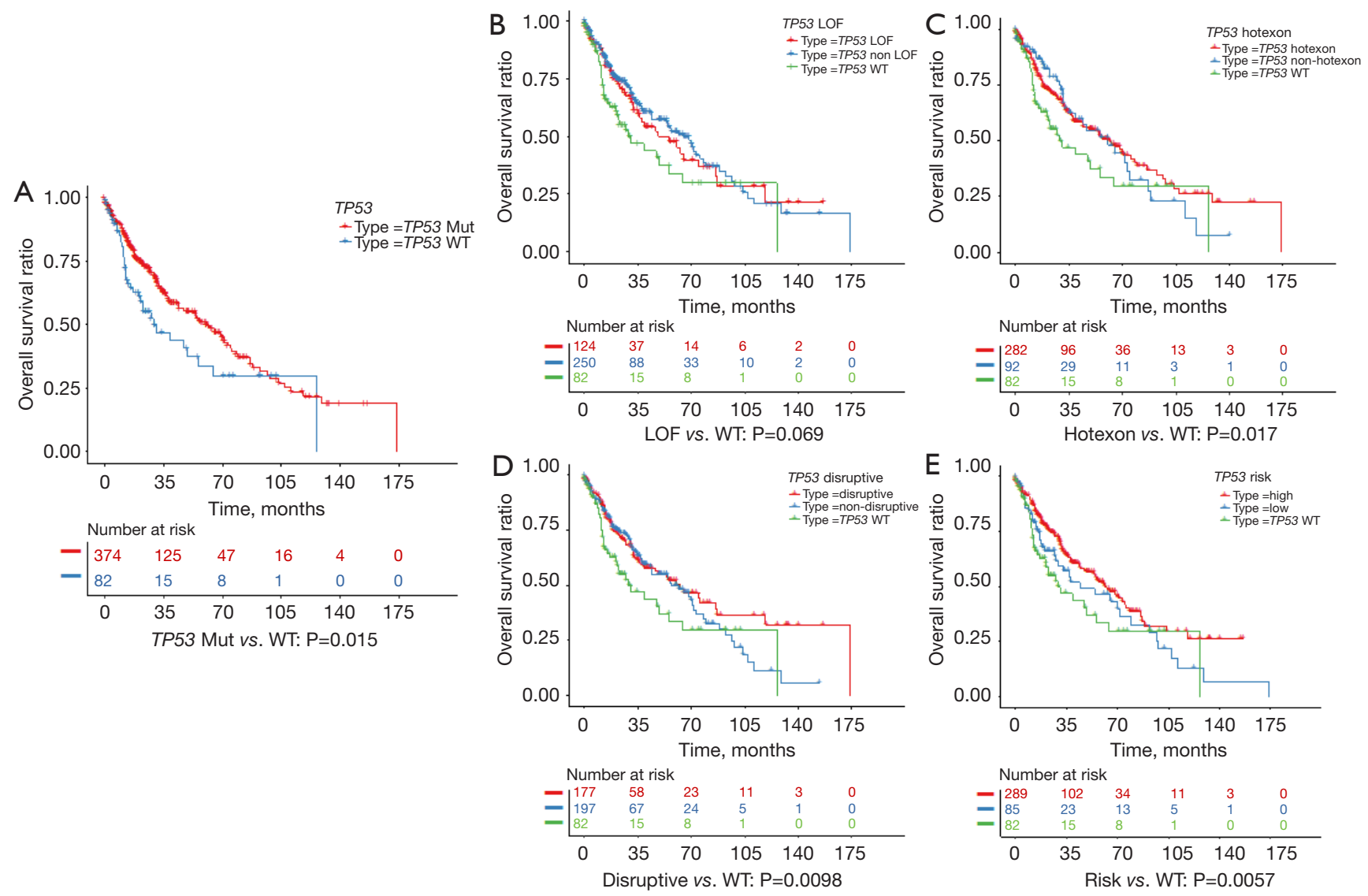

Figure 5 Kaplan-Meier survival plots showing the overall survival (OS) of patients with lung squamous cell carcinoma (LUSC), stratified by (A) TP53 mutation (TP53-MUT) status or (B-E) different classes of TP53-MUTs.

In line with this association, evidence suggests that TP53MUTs predict better OS benefited from immunotherapy in advanced NSCLC $(28,29)$. More specifically, our analysis suggested that TP53-MUTs were significantly associated with the TMB in patients with LUAD but not in those with LUSC, which had not been previously reported. However, when comparing the different classes of TP53-MUTs, the TMB was significantly higher in the hot exon group than in the non-hot exon group for patients with LUSC. This correlation between TP53-MUTs and the TMB in patients with LUSC may have prognostic or therapeutic relevance. NSCLC patients carrying TP53-MUT have longer PFS for the immunotherapy than those carrying TP53-WT (30). The different expressed genes between LUSC with TP53MUT and those with TP53-WT are closely related to immune functions indicating that genes related with TP53MUT may interfere immunotherapy (31). Hot exon type of TP53-MUT may be helpful to find out patients with LUSC who can benefit from immunotherapy, and further clinical validation is warranted.

Many researches have shown that patients with NSCLC or LUAD carrying TP53-MUT have shorter OS than those carrying TP53-WT (11,26,32-34). However, whether TP53MUTs shorten the survival of patients with LUSC is still lack of attention. Despite the similar DFS rates, patients in this study with stage I-III LUAD carrying a TP53-MUT had worse OS than those carrying the TP53-WT, although this difference did not reach statistical significance. In contrast, a TP53-MUT was a good independent prognostic factor for OS in patients with LUSC. Previous research into the prognostic impact of a TP53-MUT has focused mainly on patients with LUAD or NSCLC and seldom on those with LUSC (32-34). To the best of our knowledge, the present study is the first to show a significant association between favorable OS and TP53-MUTs in a large cohort of patients with LUSC. The opposing prognostic effects of TP53-MUTs for LUAD and LUSC suggested the p53 had different roles in the underlying biology of the 2 histologies, 


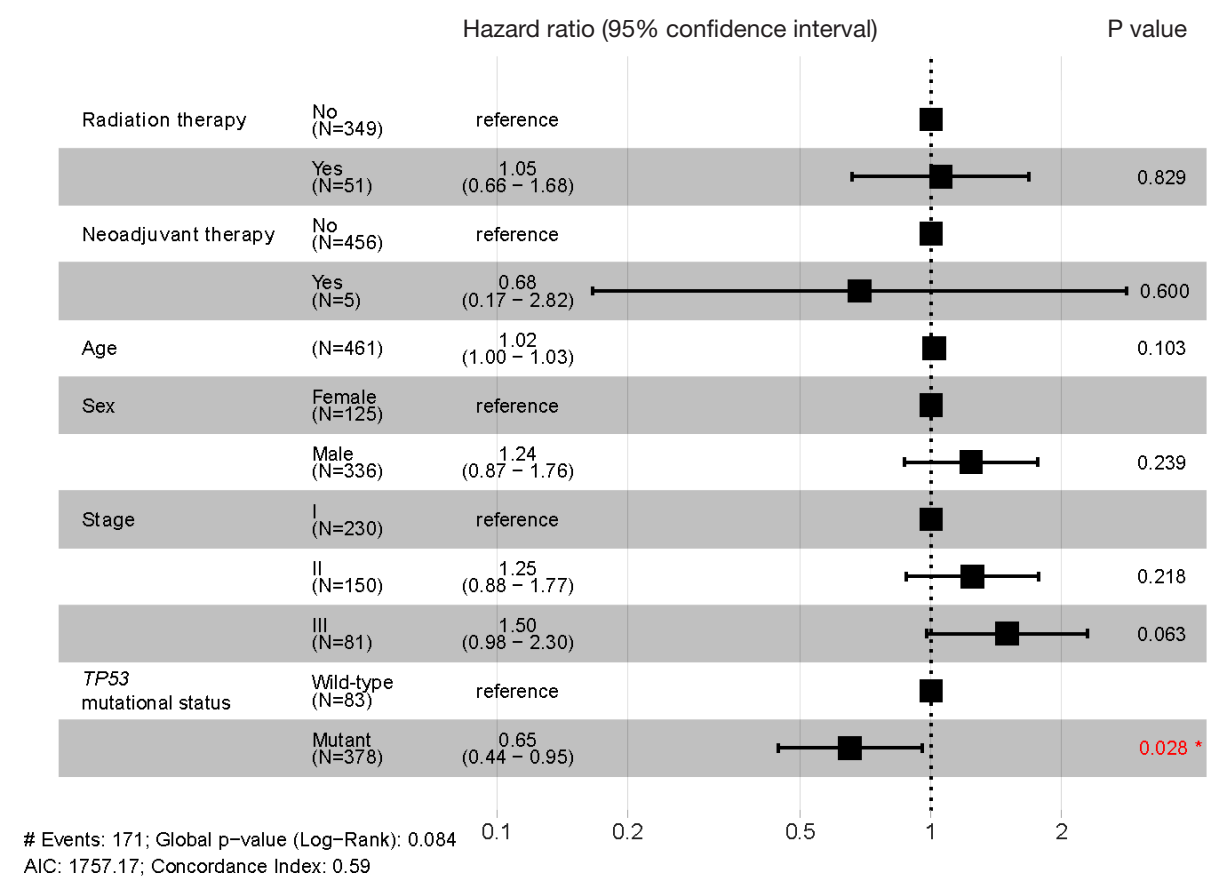

Figure 6 Multivariate analysis of prognostic factors for overall survival (OS) in patients with resectable stage I-III lung squamous cell carcinoma (LUSC) $(\mathrm{n}=461) .{ }^{*}, \mathrm{P}<0.05$.

which should be considered in the decision-making process for NSCLC treatment.

We used 4 TP53-MUT classification methods to interrogate whether TP53-MUT types could lead to further stratification among patients carrying a TP53-MUT. For patients with LUAD, none of the subgroups showed different OS compared to patients carrying the TP53-WT, whereas for patients with LUSC, carriers of non-LOF, hot exon, disruptive, or EAp53 high-risk TP53-MUTs all manifested a lower risk of death compared with patients carrying the TP53-WT. Also, the EAp53 high-risk group showed a trend toward prolonged OS compared with the EAp53 low-risk group. Although little has been reported on the comparisons between the TP53-MUT subtypes, a few studies have examined the prognostic value of different TP53-MUT types by comparing their carriers to patients carrying the TP53-WT. For instance, in patients with stage I-III NSCLC receiving platinum-containing adjuvant chemotherapy, those carrying the hot exon TP53-MUTs showed a poorer prognosis than those with TP53-WT (35). The same was observed in patients with advanced $A L K$ rearranged NSCLC receiving crizotinib, an $A L K$ TKI (36). In head and neck squamous cell carcinoma, patients in EAp53 high-risk groups have manifested poorer survival than those with TP53-WT (10). Another small-cohort study showed an association between EAp53 high-risk mutations and shorter recurrence-free survival (RFS) and OS, and an increased TMB compared with patients carrying EAp53 low-risk mutations or the TP53-WT (37). On the other hand, patients with NSCLC carrying the EAp53 low-risk TP53-MUT were also shown to have a significantly better OS rate than those carrying the EAp53 high-risk TP53-MUT or the TP53-WT (20). The median OS rate after initial diagnosis of metastasis was an impressive 64.5 months for the EAp53 lowrisk TP53-MUT (20). Furthermore, the risk of death in patients carrying the EAp53 low-risk mutation was reduced by $70 \%$ and $48 \%$ when compared to those carrying the EAp53 high-risk mutation and the TP53-WT, respectively (20). Interestingly, when KRAS mutations coexistence, the survival advantage was absent in patients carrying EAp53 low-risk mutation, which illustrated the importance of the mutational landscape in prognostication (20). In this study, we observed the consistent prognostic effects of the EAp53 scoring system and, for the first time, applied this system to the survival analysis for NSCLC subtypes. EAp53 scores may currently be the most powerful prognostication tool for stratifying 
patients carrying TP53-MUTs.

There were several limitations to our study. In this database-driven clinical investigation, we did not provide clear explanations for the underlying biological mechanisms for our findings. Also, these findings await future studies for further validation.

\section{Conclusions}

In summary, we characterized the distribution of mutation rates for various types of TP53-MUTs in patients with NSCLC, identified concurrent and mutually exclusive genomic alterations and revealed histology-specific differences in the OS of patients carrying a TP53-MUT. TP53-MUTs serve as a poor prognosis factor for patients with LUAD and a good prognosis factor for those with LUSC, raising the question of whether LUSC in patients carrying a TP53-MUT has a distinct etiology and should be considered as an atypical LUSC subtype. Findings from this study reinforce the relevance of TP53-MUTs in NSCLC prognostication and may aid the development of TP53targeted therapy and the management of operable NSCLC.

\section{Acknowledgments}

Funding: This study was supported by the Natural Science Foundation of Hebei Province (H2020206551) and the Cancer Research Program of National Cancer Center (NCC2017A30).

\section{Footnote}

Reporting Checklist: The authors have completed the REMARK reporting checklist. Available at https://atm. amegroups.com/article/view/10.21037/atm-22-412/rc

Conflicts of Interest: All authors have completed the ICMJE uniform disclosure form (available at https://atm. amegroups.com/article/view/10.21037/atm-22-412/coif). QZ was a student of Hebei medical university and studied in the Forth Hospital of Hebei medical university during the conduct of this study. XZ, YC and YS were employees of Burning Rock Biotech during the conduct of this study. The other authors have no conflicts of interest to declare.

Ethical Statement: The authors are accountable for all aspects of the work in ensuring that questions related to the accuracy or integrity of any part of the work are appropriately investigated and resolved. This study does not involve ethical and moral constraints, and the data in this article are derived from public databases. The study was conducted in accordance with the Declaration of Helsinki (as revised in 2013).

Open Access Statement: This is an Open Access article distributed in accordance with the Creative Commons Attribution-NonCommercial-NoDerivs 4.0 International License (CC BY-NC-ND 4.0), which permits the noncommercial replication and distribution of the article with the strict proviso that no changes or edits are made and the original work is properly cited (including links to both the formal publication through the relevant DOI and the license). See: https://creativecommons.org/licenses/by-nc-nd/4.0/.

\section{References}

1. Sung H, Ferlay J, Siegel RL, et al. Global Cancer Statistics 2020: GLOBOCAN Estimates of Incidence and Mortality Worldwide for 36 Cancers in 185 Countries. CA Cancer J Clin 2021;71:209-49.

2. Shugeng Gao, Ning Li, Shuhang Wang, Fan Zhang, Wenqiang Wei, Ni Li, Nan Bi, Zhijie Wang, Jie He. Lung Cancer in People's Republic of China. J Thorac Oncol. 2020;15:1567-1576.

3. Miller KD, Nogueira L, Mariotto AB, et al. Cancer treatment and survivorship statistics, 2019. CA Cancer J Clin 2019;69:363-85.

4. Huang $M$, Jin J, Zhang F, et al. Non-disruptive mutation in TP53 DNA-binding domain is a beneficial factor of esophageal squamous cell carcinoma. Ann Transl Med 2020;8:316.

5. Zhou X, Hao Q, Lu H. Mutant p53 in cancer therapy-the barrier or the path. J Mol Cell Biol 2019;11:293-305.

6. Le Calvez F, Mukeria A, Hunt JD, et al. TP53 and KRAS mutation load and types in lung cancers in relation to tobacco smoke: distinct patterns in never, former, and current smokers. Cancer Res 2005;65:5076-83.

7. Boyarskikh UA, Gulyaeva LF, Avdalyan AM, et al. Spectrum of TP53 Mutations in BRCA1/2 Associated High-Grade Serous Ovarian Cancer. Front Oncol 2020;10:1103.

8. Zhang W, Edwards A, Flemington EK, et al. Significant Prognostic Features and Patterns of Somatic TP53 Mutations in Human Cancers. Cancer Inform 2017;16:1176935117691267.

9. Poeta ML, Manola J, Goldwasser MA, et al. TP53 
mutations and survival in squamous-cell carcinoma of the head and neck. N Engl J Med 2007;357:2552-61.

10. Neskey DM, Osman AA, Ow TJ, et al. Evolutionary Action Score of TP53 Identifies High-Risk Mutations Associated with Decreased Survival and Increased Distant Metastases in Head and Neck Cancer. Cancer Res 2015;75:1527-36.

11. Mogi A, Kuwano H. TP53 mutations in nonsmall cell lung cancer. J Biomed Biotechnol 2011;2011:583929.

12. Cancer Genome Atlas Research Network. Comprehensive molecular profiling of lung adenocarcinoma. Nature 2014;511:543-50.

13. Cancer Genome Atlas Research Network. Comprehensive genomic characterization of squamous cell lung cancers. Nature 2012;489:519-25.

14. Skoulidis F, Heymach JV. Co-occurring genomic alterations in non-small-cell lung cancer biology and therapy. Nat Rev Cancer 2019;19:495-509.

15. Qin K, Hou H, Liang Y, et al. Prognostic value of TP53 concurrent mutations for EGFR-TKIs and ALK-TKIs based targeted therapy in advanced non-small cell lung cancer: a meta-analysis. BMC Cancer 2020;20:328.

16. Shajani-Yi Z, de Abreu FB, Peterson JD, et al. Frequency of Somatic TP53 Mutations in Combination with Known Pathogenic Mutations in Colon Adenocarcinoma, NonSmall Cell Lung Carcinoma, and Gliomas as Identified by Next-Generation Sequencing. Neoplasia 2018;20:256-62 .

17. Jiao XD, Qin BD, You P, et al. The prognostic value of TP53 and its correlation with EGFR mutation in advanced non-small cell lung cancer, an analysis based on cBioPortal data base. Lung Cancer 2018;123:70-5.

18. Tanimoto A, Matsumoto S, Takeuchi S, et al. Proteasome Inhibition Overcomes ALK-TKI Resistance in ALKRearranged/TP53-Mutant NSCLC via Noxa Expression. Clin Cancer Res 2021;27:1410-20.

19. Dong ZY, Zhong WZ, Zhang XC, et al. Potential Predictive Value of TP53 and KRAS Mutation Status for Response to PD-1 Blockade Immunotherapy in Lung Adenocarcinoma. Clin Cancer Res 2017;23:3012-24.

20. Wang Y, Wang Z, Piha-Paul S, et al. Outcome analysis of Phase I trial patients with metastatic KRAS and/or TP53 mutant non-small cell lung cancer. Oncotarget 2018;9:33258-70.

21. Powles T, Durán I, van der Heijden MS, et al. Atezolizumab versus chemotherapy in patients with platinum-treated locally advanced or metastatic urothelial carcinoma (IMvigor211): a multicentre, open-label, phase 3 randomised controlled trial. Lancet 2018;391:748-57.
Erratum in: Lancet 2018;392:1402.

22. Carbone DP, Reck M, Paz-Ares L, et al. First-Line Nivolumab in Stage IV or Recurrent Non-Small-Cell Lung Cancer. N Engl J Med 2017;376:2415-26.

23. Hellmann MD, Nathanson T, Rizvi H, et al. Genomic Features of Response to Combination Immunotherapy in Patients with Advanced Non-Small-Cell Lung Cancer. Cancer Cell 2018;33:843-852.e4.

24. Antonia SC, Callahan MK, Awad MM. et al. Impact of tumor mutation burden on the efficacy of nivolumab or nivolumab+ipilimumab in small cell lung cancer: an exploratory analysis of CheckMate 032 . In World Conference on Lung Cancer, 2017.

25. Offin M, Rizvi H, Tenet M, et al. Tumor Mutation Burden and Efficacy of EGFR-Tyrosine Kinase Inhibitors in Patients with EGFR-Mutant Lung Cancers. Clin Cancer Res 2019;25:1063-9.

26. Li L, Li M, Wang X. Cancer type-dependent correlations between TP53 mutations and antitumor immunity. DNA Repair (Amst) 2020;88:102785.

27. Ozaki Y, Muto S, Takagi H, et al. Tumor mutation burden and immunological, genomic, and clinicopathological factors as biomarkers for checkpoint inhibitor treatment of patients with non-small-cell lung cancer. Cancer Immunol Immunother 2020;69:127-34.

28. Biton J, Mansuet-Lupo A, Pécuchet N, et al. TP53, STK11, and EGFR Mutations Predict Tumor Immune Profile and the Response to Anti-PD-1 in Lung Adenocarcinoma. Clin Cancer Res 2018;24:5710-23.

29. Assoun S, Theou-Anton N, Nguenang M, et al. Association of TP53 mutations with response and longer survival under immune checkpoint inhibitors in advanced non-small-cell lung cancer. Lung Cancer 2019;132:65-71.

30. Wang S, Jiang M, Yang Z, et al. The role of distinct comutation patterns with TP53 mutation in immunotherapy for NSCLC. Genes Dis 2020;9:245-51.

31. $\mathrm{Xu} \mathrm{F}$, Lin H, He P, et al. A TP53-associated gene signature for prediction of prognosis and therapeutic responses in lung squamous cell carcinoma. Oncoimmunology 2020;9:1731943.

32. La Fleur L, Falk-Sörqvist E, Smeds P, et al. Mutation patterns in a population-based non-small cell lung cancer cohort and prognostic impact of concomitant mutations in KRAS and TP53 or STK11. Lung Cancer 2019;130:50-8.

33. Xu S, Wang Y, Ren F, et al. Impact of genetic alterations on outcomes of patients with stage I nonsmall cell lung cancer: An analysis of the cancer genome atlas data. Cancer Med 2020;9:7686-94. 


\section{Page 12 of 12}

34. Deben C, Van den Bossche J, Van Der Steen N, et al. Deep sequencing of the TP53 gene reveals a potential risk allele for non-small cell lung cancer and supports the negative prognostic value of TP53 variants. Tumour Biol 2017;39:1010428317694327.

35. Ma X, Le Teuff G, Lacas B, et al. Prognostic and Predictive Effect of TP53 Mutations in Patients with Non-Small Cell Lung Cancer from Adjuvant CisplatinBased Therapy Randomized Trials: A LACE-Bio Pooled Analysis. J Thorac Oncol 2016;11:850-61.

Cite this article as: Fan Z, Zhang Q, Feng L, Wang L, Zhou X, Han J, Li D, Liu J, Zhang X, Zuo J, Zou X, Cai Y, Sun Y, Wang Y. Genomic landscape and prognosis of patients with TP53-mutated non-small cell lung cancer. Ann Transl Med 2022;10(4):188. doi: 10.21037/atm-22-412

\section{Fan et al. The prognosis of NSCLC patients with TP53-mutation}

36. Wang WX, Xu CW, Chen YP, et al. TP53 mutations predict for poor survival in ALK rearrangement lung adenocarcinoma patients treated with crizotinib. J Thorac Dis 2018;10:2991-8.

37. Zhao Y, Han H, Gao Z, et al. Evolutionary Action Score of TP53 Enhances the Prognostic Prediction for Stage I Lung Adenocarcinoma. Semin Thorac Cardiovasc Surg 2021;33:221-9.

(English Language Editor: L. Roberts) 


\section{Supplementary}

A

\begin{tabular}{|c|c|c|c|c|c|c|c|c|c|c|}
\hline EGFR - & $7.3 \%$ & $17.2 \%$ & $15.5 \%$ & $16.3 \%$ & $15.4 \%$ & $16.4 \%$ & $15.8 \%$ & $15.1 \%$ & $19.1 \%$ & \\
\hline ALK - & $3.6 \%$ & $7.5 \%$ & $9.1 \%$ & $8.9 \%$ & $7.7 \%$ & $8.2 \%$ & $8.9 \%$ & $9.9 \%$ & $4.4 \%$ & \\
\hline MET - & $4.6 \%$ & $5.4 \%$ & $2.7 \%$ & $3.5 \%$ & $3.8 \%$ & $5.2 \%$ & $2.1 \%$ & $4.2 \%$ & $1.5 \%$ & \\
\hline BRAF - & $5 \%$ & $9.7 \%$ & $10.7 \%$ & $11.4 \%$ & $7.7 \%$ & $6.7 \%$ & $13.7 \%$ & $9.9 \%$ & $11.8 \%$ & \\
\hline KRAS - & $33.7 \%$ & $24.7 \%$ & $20.3 \%$ & $20.8 \%$ & $24.4 \%$ & $22.4 \%$ & $21.2 \%$ & $23.1 \%$ & $17.6 \%$ & \\
\hline ERBB2 - & $2 \%$ & $1.1 \%$ & $2.7 \%$ & $2.5 \%$ & $1.3 \%$ & $1.5 \%$ & $2.7 \%$ & $2.8 \%$ & $0 \%$ & \\
\hline ROS1- & $3.6 \%$ & $2.2 \%$ & $9.1 \%$ & $6.4 \%$ & $7.7 \%$ & $5.2 \%$ & $8.2 \%$ & $6.1 \%$ & $8.8 \%$ & \\
\hline RET - & $1 \%$ & $9.7 \%$ & $5.3 \%$ & $5.9 \%$ & $9 \%$ & $8.2 \%$ & $5.5 \%$ & $7.5 \%$ & $4.4 \%$ & \\
\hline TTN - & $28.1 \%$ & $58.1 \%$ & $63.1 \%$ & $60.4 \%$ & $64.1 \%$ & $55.2 \%$ & $67.1 \%$ & $61.3 \%$ & $61.8 \%$ & Frequency \\
\hline MUC16 - & $25.1 \%$ & $54.8 \%$ & $53.5 \%$ & $53.5 \%$ & $55.1 \%$ & $50 \%$ & $57.5 \%$ & $58 \%(0.022$ & $41.2 \%(0.022)$ & 0.6 \\
\hline RYR2- & $19.1 \%$ & $52.7 \%$ & $49.2 \%$ & $49 \%$ & $53.8 \%$ & $50 \%$ & $50.7 \%$ & $51.9 \%$ & $45.6 \%$ & \\
\hline CSMD3 - & $19.8 \%$ & $53.8 \%$ & $56.7 \%$ & $53.5 \%$ & $61.5 \%$ & $52.2 \%$ & $58.9 \%$ & $56.6 \%$ & $52.9 \%$ & 0.4 \\
\hline LRP1B - & $19.8 \%$ & $45.2 \%$ & $45.5 \%$ & $43.6 \%$ & $50 \%$ & $43.3 \%$ & $47.3 \%$ & $47.6 \%$ & $38.2 \%$ & 0.2 \\
\hline USH2A - & $17.8 \%$ & $41.9 \%$ & $41.2 \%$ & $38.6 \%$ & $48.7 \%$ & $41 \%$ & $41.8 \%$ & $42.5 \%$ & $38.2 \%$ & 0.0 \\
\hline $\mathrm{ZFHX4}-$ & $17.8 \%$ & $48.4 \%$ & $40.6 \%$ & $42.6 \%$ & $44.9 \%$ & $43.3 \%$ & $43.2 \%$ & $46.2 \%$ & $33.8 \%$ & \\
\hline XIRP2 - & $13.2 \%$ & $29 \%$ & $34.8 \%$ & $34.7 \%$ & $28.2 \%$ & $27.6 \%$ & $37.7 \%$ & $33.5 \%$ & $30.9 \%$ & \\
\hline SPTA1 - & $15.2 \%$ & $33.3 \%$ & $33.7 \%$ & $33.2 \%$ & $34.6 \%$ & $30.6 \%$ & $36.3 \%$ & $35.4 \%$ & $27.9 \%$ & \\
\hline FLG - & $14.2 \%$ & $40.9 \%$ & $28.9 \%$ & $29.2 \%$ & $42.3 \%$ & $36.6 \%$ & $29.5 \%$ & $35.4 \%$ & $25 \%$ & \\
\hline FАTЗ - & $10.9 \%$ & $21.5 \%$ & $31.6 \%$ & $29.2 \%$ & $25.6 \%$ & $22.4 \%$ & $33.6 \%$ & $28.8 \%$ & $26.5 \%$ & \\
\hline CSMD1 - & $15.5 \%$ & $25.8 \%$ & $23 \%$ & $24.3 \%$ & $23.1 \%$ & $24.6 \%$ & $23.3 \%$ & $25.5 \%$ & $19.1 \%$ & \\
\hline ZNF536 - & $9.9 \%$ & $25.8 \%$ & $30.5 \%$ & $28.2 \%$ & $30.8 \%$ & $27.6 \%$ & $30.1 \%$ & $29.2 \%$ & $27.9 \%$ & \\
\hline RYR3 - & $9.6 \%$ & $28 \%$ & $23.5 \%$ & $23.8 \%$ & $28.2 \%$ & $25.4 \%$ & $24.7 \%$ & $25.5 \%$ & $23.5 \%$ & \\
\hline & & & & & & & & $(40)^{n}$ & $v^{\circ}$ & \\
\hline
\end{tabular}

B

\begin{tabular}{|c|c|c|c|c|c|c|c|c|c|c|}
\hline TTN - & $44.6 \%$ & $74.5 \%$ & $77.8 \%$ & $77.1 \%$ & $75.7 \%$ & $73.9 \%$ & $79.2 \%$ & $74.2 \%$ & & \multirow{26}{*}{$\begin{array}{l}\text { Frequency } \\
-0.8\end{array}$} \\
\hline Muc16- & $28.9 \%$ & $38 \%$ & $42.3 \%$ & $41.3 \%$ & $39.8 \%$ & $44.2 \%$ & $38.1 \%$ & $41.3 \%$ & $39.6 \%$ & \\
\hline CSMD3 - & $21.7 \%$ & $48.9 \%$ & $48.1 \%$ & $48.9 \%$ & $46.6 \%$ & $47.2 \%$ & $49.4 \%$ & $47.1 \%$ & $52.5 \%$ & \\
\hline RYR2- & $26.5 \%$ & $38.7 \%$ & $44.4 \%$ & $42.5 \%$ & $42.7 \%$ & $42.2 \%$ & $42.9 \%$ & $42.6 \%$ & $42.6 \%$ & \\
\hline LRP1B - & $18.1 \%$ & $35 \%$ & $40.6 \%$ & $41.6 \%(0.049)$ & $30.1 \%(0.049)$ & $38.2 \%$ & $39.4 \%$ & $38.9 \%$ & $38.6 \%$ & \\
\hline SYNE1 - & $24.1 \%$ & $28.5 \%$ & $33.4 \%$ & $34.6 \%(0.044)$ & $23.3 \%(0.044)$ & $29.6 \%$ & $33.8 \%$ & $31 \%$ & $34.7 \%$ & \\
\hline USH2A- & $22.9 \%$ & $29.2 \%$ & $38.2 \%$ & $37.9 \%$ & $27.2 \%$ & $28.6 \%(0.009)$ & $41.1 \%(0.009)$ & $32.5 \%(0.036)$ & $44.6 \%(0.036)$ & \\
\hline ZFHX4- & $18.1 \%$ & $31.4 \%$ & $31.7 \%$ & $33 \%$ & $27.2 \%$ & $32.7 \%$ & $30.7 \%$ & $30.1 \%$ & $36.6 \%$ & \\
\hline FAM135B - & $8.4 \%$ & $25.5 \%$ & $28 \%$ & $27.8 \%$ & $25.2 \%$ & $28.6 \%$ & $26 \%$ & $28 \%$ & $24.8 \%$ & \\
\hline NAV3- & $15.7 \%$ & $20.4 \%$ & $22.9 \%$ & $23.2 \%$ & $18.4 \%$ & $20.1 \%$ & $23.8 \%$ & $20.7 \%$ & $26.7 \%$ & \\
\hline XIRP2 - & $14.5 \%$ & $17.5 \%$ & $21.8 \%$ & $21.1 \%$ & $18.4 \%$ & $20.6 \%$ & $20.3 \%$ & $18.8 \%$ & $25.7 \%$ & \\
\hline $\mathrm{CDH} 10-$ & $12 \%$ & $19 \%$ & $22.9 \%$ & $21.4 \%$ & $22.3 \%$ & $23.6 \%$ & $19.9 \%$ & $22.8 \%$ & $17.8 \%$ & \\
\hline SPTA1 - & $12 \%$ & $21.9 \%$ & $22.2 \%$ & $23.9 \%$ & $16.5 \%$ & $19.6 \%$ & $24.2 \%$ & $21 \%$ & $25.7 \%$ & \\
\hline DNAH5 - & $8.4 \%$ & $16.8 \%$ & $23.9 \%$ & $22.3 \%$ & $19.4 \%$ & $19.1 \%$ & $23.8 \%$ & $21 \%$ & $23.8 \%$ & \\
\hline КмT2D - & $16.9 \%$ & $30.7 \%$ & $21.8 \%$ & $26.3 \%$ & $19.4 \%$ & $27.6 \%$ & $22.1 \%$ & $23.7 \%$ & $27.7 \%$ & \\
\hline RYR3 - & $10.8 \%$ & $19 \%$ & $24.6 \%$ & $22.9 \%$ & $22.3 \%$ & $22.1 \%$ & $23.4 \%$ & $22.2 \%$ & $24.8 \%$ & \\
\hline PAPPA2- & $19.3 \%$ & $16.1 \%$ & $18.1 \%$ & $18.7 \%$ & $13.6 \%$ & $18.1 \%$ & $16.9 \%$ & $17.6 \%$ & $16.8 \%$ & \\
\hline PKHD1L1 - & $10.8 \%$ & $21.2 \%$ & $21.2 \%$ & $21.1 \%$ & $21.4 \%$ & $20.1 \%$ & $22.1 \%$ & $21 \%$ & $21.8 \%$ & \\
\hline PKHD1 - & $8.4 \%$ & $19.7 \%$ & $21.2 \%$ & $21.1 \%$ & $19.4 \%$ & $21.1 \%$ & $20.3 \%$ & $20.1 \%$ & $22.8 \%$ & \\
\hline PCLO- & $14.5 \%$ & $17.5 \%$ & $19.8 \%$ & $19.9 \%$ & $16.5 \%$ & $19.6 \%$ & $18.6 \%$ & $20.1 \%$ & $15.8 \%$ & \\
\hline PCDH15- & $16.9 \%$ & $16.8 \%$ & $21.5 \%$ & $21.1 \%$ & $16.5 \%$ & $18.6 \%$ & $21.2 \%$ & $18.2 \%$ & $25.7 \%$ & \\
\hline MUC17- & $12 \%$ & $19.7 \%$ & $16 \%$ & $16.2 \%$ & $20.4 \%$ & $18.6 \%$ & $16 \%$ & $18.2 \%$ & $13.9 \%$ & \\
\hline FAT3 - & $10.8 \%$ & $13.9 \%$ & $20.8 \%$ & $19.3 \%$ & $16.5 \%$ & $15.6 \%$ & $21.2 \%$ & $16.7 \%$ & $24.8 \%$ & \\
\hline ERICH3 - & $7.2 \%$ & $21.2 \%$ & $19.5 \%$ & $20.2 \%$ & $19.4 \%$ & $21.6 \%$ & $18.6 \%$ & $19.5 \%$ & $21.8 \%$ & \\
\hline FLG - & $12 \%$ & $15.3 \%$ & $20.1 \%$ & $20.5 \%$ & $12.6 \%$ & $19.1 \%$ & $18.2 \%$ & $18.8 \%$ & $17.8 \%$ & \\
\hline & & & & & & & 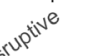 & (rig & $\nu^{O^{N}}$ & \\
\hline
\end{tabular}

Figure S1 Mutation rates of concomitant genomic alterations in carriers of different types of TP53 mutations (TP53-MUTs) among (A) patients with lung adenocarcinoma (LUAD) or (B) patients with lung squamous cell carcinoma (LUSC). 

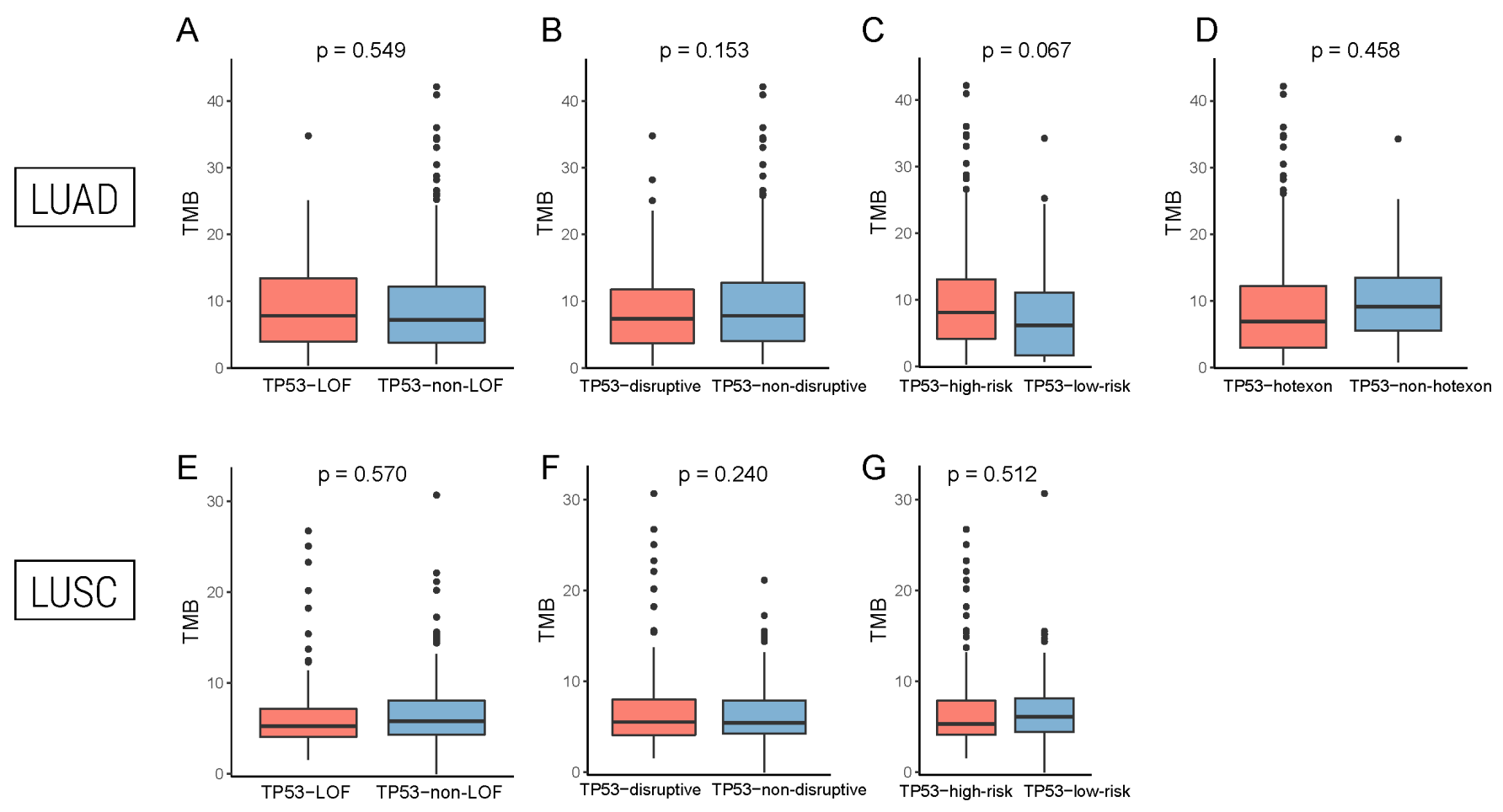

Figure S2 The tumor mutation burden (TMB) levels of (A-D) patients with lung adenocarcinoma (LUAD) or (E-G) those with squamous cell carcinoma (LUSC) carrying the indicated subtypes of TP53 mutations (TP53-MUTs). 
A

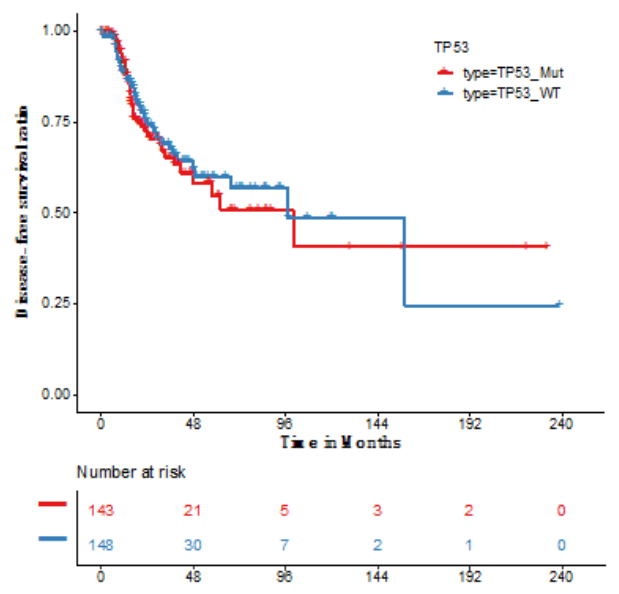

TP53 Mut VS WT : P= 0.64
B

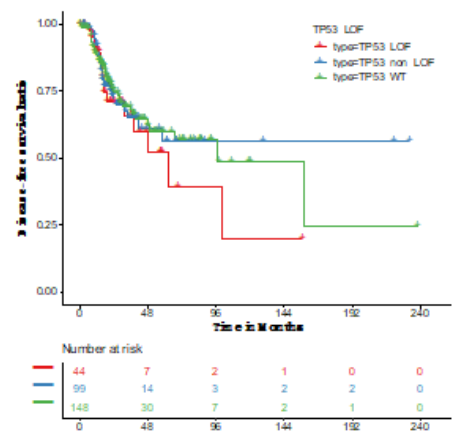

LOF VS WT : $P=0.37$

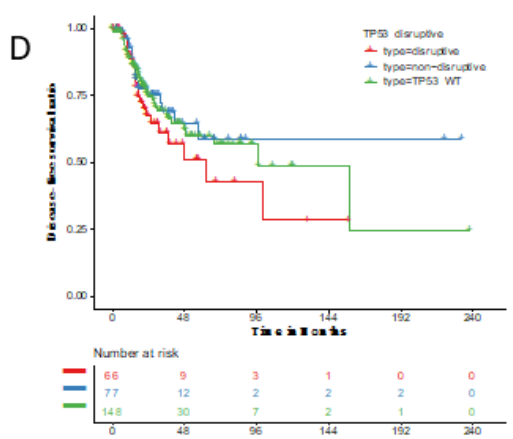

Disruptive VS WT : $\mathrm{P}=0.25$
C
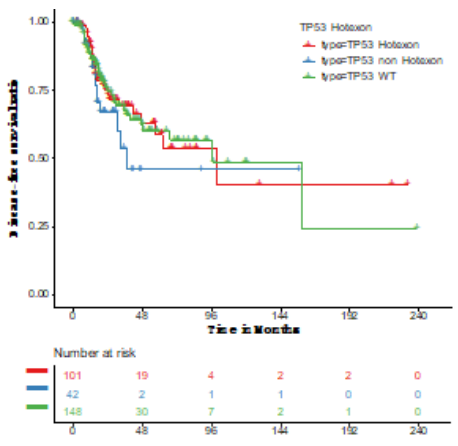

Hotexon VS WT : P $=0.98$

E

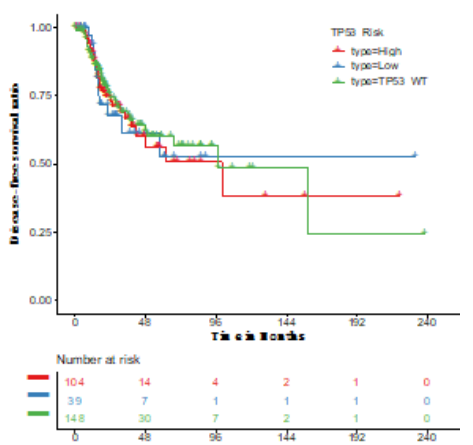

Risk VS WT : $P=0.7$

Figure S3 Kaplan-Meier survival plots showing the disease-free survival (DFS) rates of patients with lung adenocarcinoma (LAUD), stratified by (A) TP53 mutation (TP53-MUT) status or (B-E) different classes of TP53-MUTSs. 
A

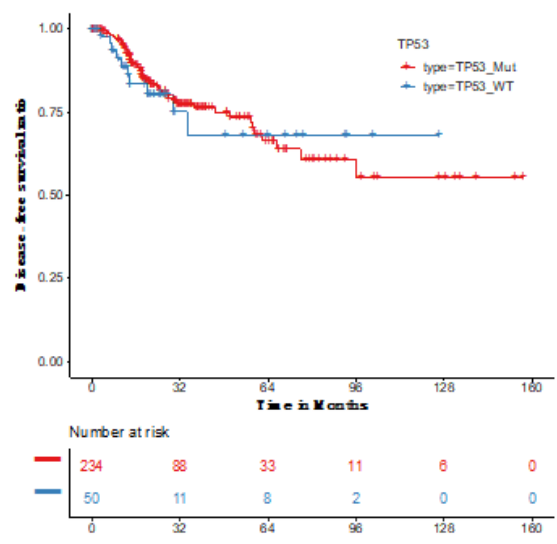

TP53 Mut VS WT : $P=0.8$
B

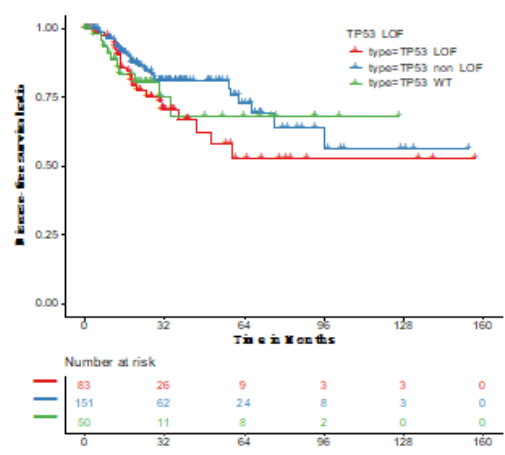

LOF VS WT : $\mathrm{P}=0.58$

D

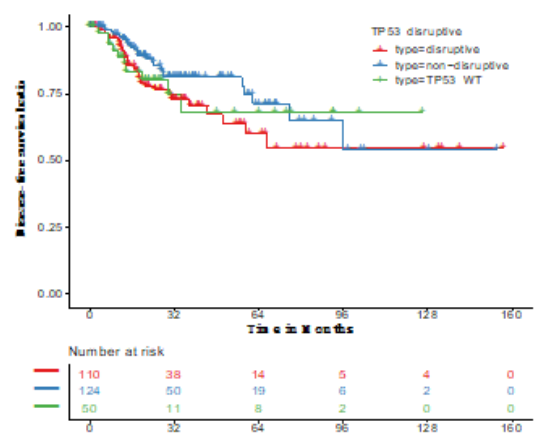

Hotexon VS WT : $\mathrm{P}=0.68$

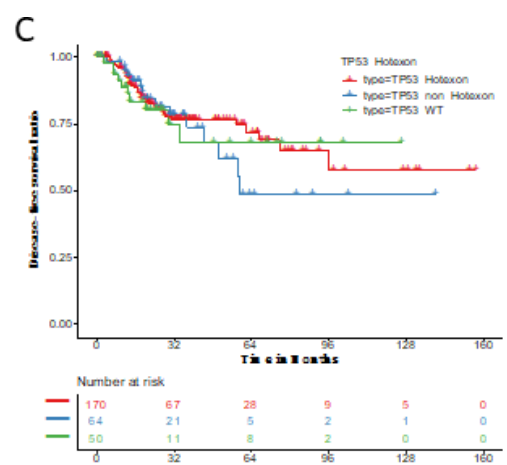

Disruptive VS WT : $\mathrm{P}=0.71$

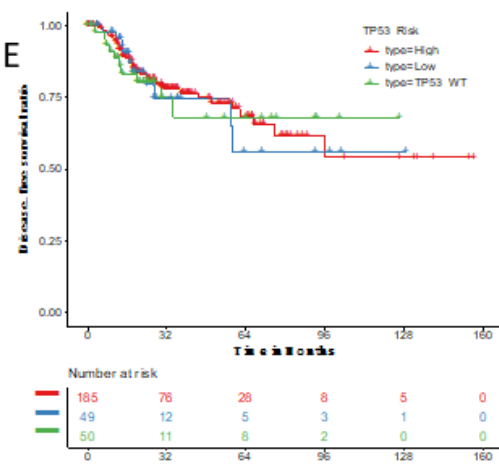

Risk VS WT : $\mathrm{P}=0.76$

Figure S4 Kaplan-Meier survival plots showing the disease-free survival (DFS) rates of patients with lung squamous cell carcinoma (LUSC), stratified by (A) TP53 mutation (TP53-MUT) status or (B-E) different classes of TP53-MUTs.

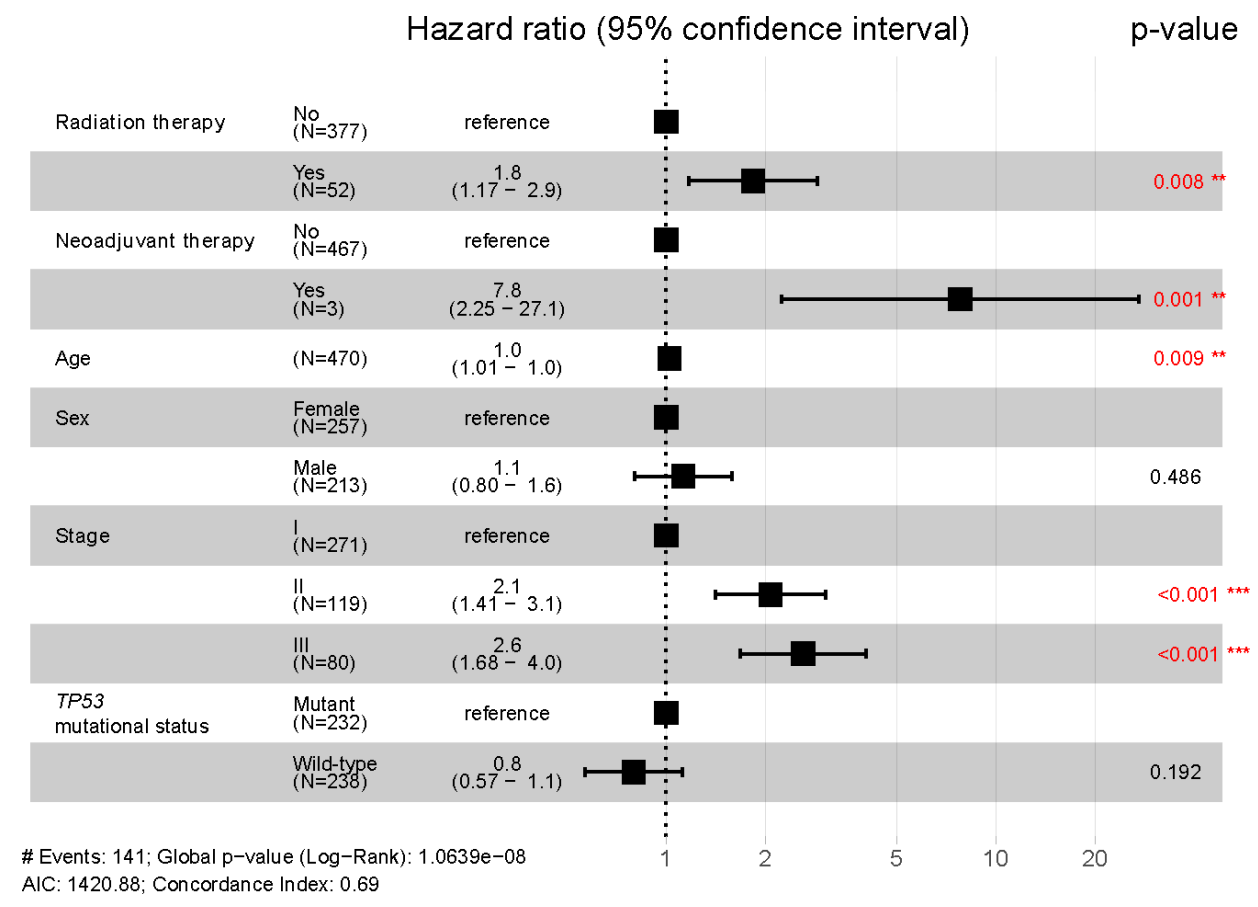

Figure S5 Multivariate analysis of prognostic factors for overall survival (OS) in patients with resectable stage I-III lung adenocarcinoma $(\operatorname{LAUD})(\mathrm{n}=470) .{ }^{* *}, \mathrm{P}<0.01 ;{ }^{* * *}, \mathrm{P}<0.001$ 